\title{
An Evaluation Model for Web-based 3D Mass Customization Toolkit Design
}

\author{
Huiwen Zhao, Leigh McLoughlin, Valery Adzhiev, and Alexander Pasko
}

\begin{abstract}
The development of geometric modelling technologies and web technologies provides the ability to present a virtual 3D product in a mass customization (MC) toolkit. Compared with 2D graphic toolkits, 3D toolkit design requires better consideration of individual customer needs, consumer and toolkit interaction, and also a means of integrating with the underlying technical infrastructure. However, there is currently no widely accepted model or criteria to regulate and evaluate 3D MC toolkit design. Given these considerations, in this paper we provide an evaluation model for web-based 3D toolkits and a heuristic evaluation of two representative commercial web-based $3 \mathrm{D}$ toolkits. The evaluation results indicate the usefulness and effectiveness of the model as a scale for evaluating $3 \mathrm{D}$ toolkits. It also reveals that despite a fair amount of effort that has been devoted to theoretical research, current 3D toolkits are still at an early development stage. We therefore conclude this paper by identifying and encouraging further topics and questions as directions for future research.
\end{abstract}

Keywords Mass customization toolkit - 3D toolkit design · Evaluation model · Interaction design $\cdot 3 \mathrm{D}$ modelling $\cdot$ Heuristic evaluation

\section{Introduction}

The key significance of mass customization (MC) is to provide personalized service and products to meet each consumer's needs and desires [7, 29, 37]. To achieve this, consumers are allowed to take part in activities and processes which used to The original version of this chapter was revised. An erratum to this chapter can be found at https://
doi.org/10.1007/978-3-319-77556-2_44

This work is funded by the European Commission Horizon 2020 - Marie Curie Individual Fellowship [grant number 661501].

H. Zhao $(\bowtie) \cdot$ L. McLoughlin · V. Adzhiev · A. Pasko

Bournemouth University, Bournemouth, UK

e-mail: hzhao@bournemouth.ac.uk; lmcloughlin@bournemouth.ac.uk;

vadzhiev@bournemouth.ac.uk; apasko@bournemouth.ac.uk

(C) The Author(s) 2018

S. Hankammer et al. (eds.), Customization 4.0, Springer Proceedings in Business and Economics, https://doi.org/10.1007/978-3-319-77556-2_23 
be controlled by the companies [47]. Toolkits therefore have been widely used in industry as a medium between consumers and manufacturers. Since powerful computers, high-speed Internet and sophisticated web browsers facilitate the efficiency of developing customized products, most toolkits nowadays are computer-based and especially web-based. They allow consumers to design their own products or service by trial-and-error experimentation and also deliver immediate feedback of the potential outcome of their design ideas [17, 29, 34].

More importantly, the development of geometric modelling provides the means of presenting a virtual 3D product in a mass customization toolkit. Compared with 2D graphic toolkits, 3D toolkits stimulate a 3D product environment that can be interacted with in a seemingly real or physical approach. Therefore, 3D toolkits provide a more direct interaction experience. Consumers can zoom in/out and rotate $3 \mathrm{D}$ models to envision the final products $[7,26]$. Instead of clicking buttons or moving sliders to customize the product, in certain systems consumers can even directly manipulate 3D models, which gives them a better sense of control. Daugherty et al. [6] claimed that consumers' experience in a $3 \mathrm{D}$ environment is similar to examining the real product in a shop. Other researchers [26, 30, 31, 46] agreed that $3 \mathrm{D}$ toolkits create a more satisfying experience for consumers than $2 \mathrm{D}$ graphic toolkits, which then helps to increase the propensity of purchase. Therefore, $3 \mathrm{D}$ toolkits have been considered as the trend of design communication between consumers and manufacturers [22].

However, the adoption of 3D MC toolkits is still at an early stage. Compared with 2D graphic toolkits, 3D toolkit design requires "greater understanding of customer needs, design options and 3D image representation” [26]. A number of studies showed that poor 3D visualization leads to a higher chance of disorientation or motion sickness [30, 31, 46]. In addition, consumers may have difficulties in understanding 3D virtual models [15]. To achieve effective mass customization, it is therefore vitally important that the $3 \mathrm{D}$ toolkits are designed to elicit a satisfying consumer experience.

Given these considerations, this paper aims at designing an evaluation model to assess 3D MC toolkits. In working towards this goal, a literature review is presented in the following section, covering different aspects of 3D toolkit design. Based on the literature review, an evaluation model is proposed, and two 3D MC toolkits are evaluated using this model. In conclusion, significant research findings are presented, and new research questions are identified.

\section{Literature Review}

The primary task of a mass customization toolkit is to help consumers as they design or modify a product or service to meet their needs and requirements. To achieve that, it is important to offer consumers appropriate customization options. Solution space, which is understood as all the possible designs a toolkit can provide, has been considered as a determinant factor for designing an effective toolkit [15, 17]. 


\subsection{Solution Space}

Solution space decides the design freedom that a toolkit can offer to a consumer. Typically there are two elements related to the design of the solution space: the type of options it provides and the size of the choice set.

Type of Options The type of options is largely determined by the way that a product can be customized. Hermans [15] categorized four different mechanisms for customization: veneer, modularity, parametric and generative. Veneer customization allows consumers to customize products by adding a visual decorative layer to a product. This has been considered as the most common method of offering customization. The possible options for this mechanism include a list of different texts, graphics, patterns or colours which can be added to the surface of the product by processes such as printing, engraving, etching or embroidery. Modularity customization decomposes products into a set of discrete modules and options for their assembly into a customized design are presented to the user. The individual options in this approach usually feature different component designs or functions. Parametric customization is widely used in 3D toolkits, which allows consumers to customize a product by changing specific parametric values which then change the nature of the product in some way. Generative customization creates 2D or 3D forms based on an algorithm or computer programming. For example, consumers can sketch the side view of a chair and extrude it into a 3D model.

In addition to offering different types of options by considering the method of customization, a number of researchers focused on the functional and aesthetic options offered by MC systems and found functional choices require less spontaneous elaboration than aesthetic choices and aesthetic choices tend to be easier to imagine and to elaborate than functional choices [38]. They suggest providing consumers with aesthetic choices should be the main approach to mass customization, because consumers enjoy themselves more when they customize aesthetic features than functional features, regardless of individual ability differences.

Size of Solution Space The size of the solution space is the range of unique final products that a toolkit can produce. It is determined by two factors: the number of attributes for customization and the variety of values given by each attribute. However, the ideal size of the solution space is still a matter of debate. On one hand, results of empirical studies have shown that consumers are actually disappointed by the limited choices offered by most existing toolkits [26]. The majority of consumers would like to have more and a larger variety of choices and also play a more active role in the design of products $[11,12,27]$. On the other hand though, it has been found that consumers can be overwhelmed by the number of choices provided by manufacturers, which may lead to "mass confusion" [10, 28, 43]. Hunt et al. [20] found that the relationship between consumer satisfaction and the number of choices can be demonstrated in an inverted U-shaped fashion, i.e. after reaching a certain point, the more choices provided, the less satisfied consumers are. Another proposed 
approach to this problem is to convert the number of choices into the number of products in a bricks-and-mortar store to get an adequate understanding of how many choices the customer has [10].

\subsection{Interaction Design}

For online customization, the process of customizing a product is fundamentally a process of human-computer interaction. The quality of the interaction is crucial for the success of mass customization as it determines whether or not the consumer will be able to complete the customization task [41].

Gerber and Martin [14] suggest that the customization task should be set up step by step as a series of smaller tasks with increasing challenge which helps consumers to avoid getting bored or getting confused. Meanwhile, multiple pathways should be provided to allow consumers to choose their own progression, which helps to give them feelings of autonomy, ownership and control over their creation process [45]. This suggestion has been echoed by [26] which agreed that products which require consumer creativity should be customized in a flexible design procedure. However, for products that require more functionality customization, a top-down hierarchical approach should be used where general features of product functionality are selected prior to detailed design features [25]. Regardless of which approach a toolkit design follows, von Hippel [42] suggests that providing consumers with enough information about the design procedure to let them know which step they are currently in and how many steps there are until completion is very necessary.

In addition, a usable toolkit should provide consumers with clear and proper guidance through all stages of the customization process. For example, a "how-to" video tutorial, a help menu and clickable paths to further explanations can be used as support resources for consumer [14]. In order to encourage creativity, libraries of standard modules should be provided to consumers to help them focus on those aspects of their design that are truly novel, and also a preset design at the starting point is useful to make the process more accessible for consumers [16, 17, 34].

As the Internet has prompted a "participatory culture", the collaboration between consumers helps to foster joint creativity and problem-solving as well as reduce the perception of risk [34]. Therefore, it is useful to provide an online community, such as a chat room, for instant communication, and space for consumers to leave comments or to assess contributions from each other [14, 34]. In addition, a historical record of work and progress of consumer collaboration around a shared interest helps to guide and inspire further product developments [14].

$3 \mathrm{D}$ toolkits are more effective and satisfying than 2D toolkits to some extent because 3D toolkits allow consumers to directly manipulate on the virtual model of the products [26, 30, 31]. Direct manipulation includes the application of real-world metaphors to make the interaction easier for consumers to learn. In addition, the rapid feedback allows consumers to see the results of their actions, therefore giving them a sense of control and strengthening their beliefs about their ability to take further interactions [2]. 


\subsection{Enabling Technologies: 3D Modelling and Web Technologies}

Toolkit design is closely related to technology development. Specifically, 3D modelling and web technologies are the two key enabling technologies for 3D toolkit design and consumer interaction. A variety of 3D modelling technologies and web technologies has been developed, and capabilities of different technologies ultimately dictate what is possible in terms of both the solution space and also the interaction techniques that are available. It is therefore important to consider this layer in order to fully evaluate the capabilities of the MC system.

3D Modelling Technologies The 3D modelling technologies are those which describe the physical shape of the product [18]. This is a virtual representation that is originally defined by the product designer and must be adjustable by the consumer through the interaction design method. 3D modelling technologies are often known as "shape modelling" in the area of computer graphics, which has been used as a generic term for geometric modelling embracing various approaches to representing 3D products. At present, the discipline of shape modelling is in transition from an established design paradigm to a new one, and this is driven by the fundamental requirements that $\mathrm{MC}$ rely upon.

The main principle of $\mathrm{MC}$ is that the user changes a product through an interactive process. In a 3D MC approach, as the user interacts with the system, the actual shape of the product changes in some way. For this to be possible, the modelling technology has to define not just a single shape but a whole family of shapes. The ways in which the shape is changed from one to another define the interaction design possibilities, and the range of valid shapes defines the solution space.

The method of using adjustable values within a geometric model to change its shape is known as parameterization and is essential in interactive modelling based on user-modifiable definitions [39] and in optimizing shapes to satisfy some design criteria [5]. Mathematical, algorithmic, and software support for defining a parametric family of shapes, such that each new set of parameter values corresponds to another valid instance of a shape, is one of the ultimate goals of shape modelling research and development. It is this parameterization of the model that makes full 3D MC possible, and different methods of parameterization offer different interaction types and different solution spaces. Further, a professional designer and a non-professional consumer have to deal with the same parameterized model using different levels of access to it. For example, a professional designer can decide which parameters consumers can customize, while consumers can only interact with the parameters that the designer selects.

Enabling Web Technologies For the purpose of web-based MC, the consumer needs to interface with the 3D model, to view it and customize the product. Full CAD software is not available or suitable for this application, so lighter weight 
solutions are needed that run from a web browser. The basic tools available within a web-based environment are HTML and JavaScript scripting language [40]. Some special purpose functionality can be achieved through implementing a small application called an applet which is sent together with the web page to the user and can typically be implemented in the Java language [3]. To handle full interactive 3D graphics, the toolkit needs to employ HTML5 and WebGL [33].

These technologies will allow the user to interact with a local representation, where the 3D object and means of interaction are based in their web browser. In some cases there can be a high computational requirement, especially when modifying the model using a more powerful representation scheme. In such cases it is likely that the local machine, which could be a portable device such as a smartphone or tablet, is insufficient to handle the requests. Here, some or all computations may be handled by a server that the website contacts in the background to perform the heavier computations [1].

\subsection{Individual Differences}

Toolkit design is a complex topic that is primarily concerned with creating a satisfying consumer experience. However, every consumer is individual and unique, each with their own skills, aesthetic tastes and physical requirements. In order to create a toolkit that satisfies each consumer, the design of a toolkit must account for these individual differences $[8,13,29,38]$.

[9] found that customers who have strong insights into their own preferences and who know what they want tend to enjoy the process more than customers who lack this preference insight. Lin et al. [21] proposed three variables which are associated with individual differences and consumers' perceived value of mass customized products, namely, a need for optimization, a need for uniqueness and centrality of visual product aesthetics. They found that consumers with a higher need for optimization, a higher need for uniqueness and a higher proclivity towards aesthetics tended to be more satisfied with the final product.

In addition, toolkit design should be concerned with individual differences in knowledge, skills, creative talent and even previous experience in mass customization [4, 8, 13, 29, 38]. For consumers who are highly knowledgeable about customizing products, a complex toolkit that provides them with a large number of options is better suited. Similarly, consumers with more Internet experience prefer more substantive features in a toolkit to those with less experience [4]. In comparison, for less experienced consumers, more guides through the configuration process are necessary, and the size of the solution space should be limited to a few customization possibilities. Salvador et al. [36] claimed that consumers who have greater expertise in a product domain are better served by a parameter-based interface, whereas lower-expertise consumers are better served by a need-based interface. Von Hippel [42] argued a programming interface is more efficient for 
experts in computer technology, and professional designers who offer original designs for customization should be provided with a different interface and a set of different tools within the same toolkit when compared to ordinary consumers who codesign the final products.

\section{A Model for Evaluating Web-Based 3D Mass Customization Toolkits}

Based on the literature review, we propose a framework - an online 3D mass customization toolkit evaluation model - to evaluate online 3D mass customization toolkits with the focus on consumer experience as well as the underlying technical supports and their influence on the design of 3D toolkits (Fig. 1).

The centre of the evaluation model is the toolkit design. This is driven by the underlying technologies, which offer features or limitations to the toolkit design. The interaction design defines the process of customization, while the solution space provides the design possibilities. In addition, individual differences are also an important factor to evaluate because they help to create a satisfying experience for different consumers. In this sense, the evaluation model has been designed from four perspectives: solution space design, interaction design, supporting technologies (i.e. 3D modelling technologies and web technologies) and individual differences.

\subsection{Solution Space Design}

The solution space in this model is used to evaluate how diverse and how large the solution space is, i.e. the variety and quantity of potential designs a toolkit can provide. It is concerned with two aspects: the types of customization options

Fig. 1 Online 3D mass customization toolkit evaluation model

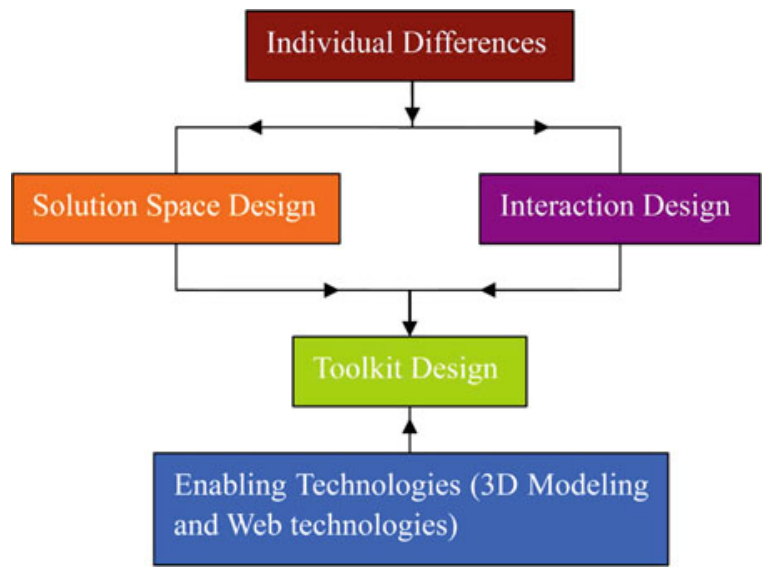


a toolkit provides and the size of the solution space. The types of options could be veneered, modular, parametric or generative alternatives when considering the approach to mass customization and then either functional or aesthetic options when considering the purpose of the product changes. The size of the solution space is usually represented by the number of potential final designs a toolkit can provide.

\subsection{Interaction Design}

The interaction design criteria here represent the evaluation of a comprehensive process. It does not only include interactions between consumers and the toolkit but also refers to interactions between consumers as well as interactions between consumers and manufacturers. Further, interaction design does not only consider the process of consumer participation but also considers how a toolkit should respond and adjust itself to consumer participation. The criteria for evaluating the interaction design are listed in Table 1.

\subsection{D Modelling and Web Technologies}

The evaluation criteria from the 3D modelling technology lead on from the literature review and are broken down into four evaluation categories:

- The representation modelling scheme. These can give us insight into what features are offered to the toolkit design.

- The level of the model parameterization. This directly enables the toolkit to offer both interaction design capabilities and the existence of the solution space. The level of model parameterization can vary from a very basic level with simple transformations (such as scale, rotation) to a high level with support for radical changes to the shape of the product (changing the actual shape, adding holes, additional geometry, etc.). The level of parameterization is closely connected with the representation scheme.

- The level of designer's support for modelling and uploading new designs. This shows how much effort a designer applies to produce a new parameterized model and to make it available to customers. For example, generative modelling for boundary representations typically requires programming a new applet or plugin to produce a design instance for the given set of parameters. On the other hand, function representation allows for a simple save and uploads procedure as the representation itself supports a high parameterization level.

- Required web technologies. Here we distinguish between basic toolkits with HTML and JavaScript implementations, advanced with Java applets and highly 3D interactive with HTML5 and WebGL employed. The required technologies can influence how the site can be viewed (e.g. on smartphone/tablet or PC) which can further affect the consumer experience. 


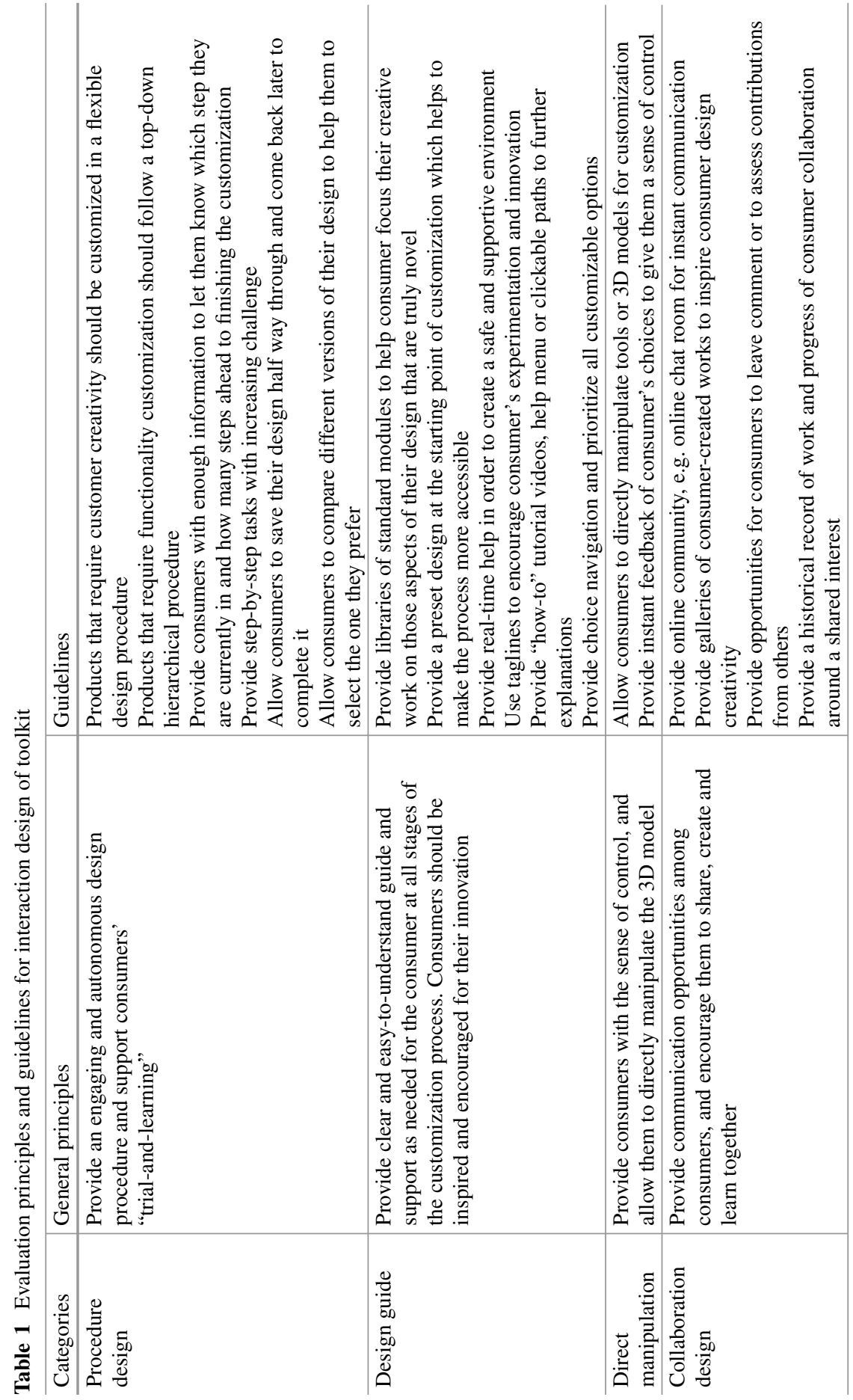




\subsection{Individual Differences}

Individual differences here are used to assess the ability of a toolkit to consider each consumer's differences in previous experience, knowledge, ability and skills. Typically, an adaptive approach for toolkit design is required in order to design a toolkit tailored to each consumer's differences. This can be achieved through three approaches:

- Provide different starting points by assessing consumer's previous experience of using the toolkit - the toolkit can "remember" previous difficulties or successes for consumers to complete a task and modify the solution space and interaction design based on the frequency and success of previous attempts.

- Provide multiple pathways to achieving a task - consumers should be able to choose their own pathway through to customize a product.

- Provide assortment matching, i.e. automatically recommend configurations for consumers by matching their needs with characteristics of existing solution spaces [37].

\section{A Heuristic Evaluation of Online 3D Toolkits}

In order to better understand the proposed evaluation model for online 3D MC toolkit and the specific principles developed in this study, a heuristic evaluation was applied to two online 3D MC toolkits design: Sandboxr ${ }^{1}$ and Nervous System ${ }^{2}$.

A heuristic evaluation is an inspection technique which aims at identifying usability problems associated with the design of user interfaces by applying a set of predefined evaluation principles [32]. A number of studies also confirmed that heuristic evaluation helps designers to find important classes of problems that are not always found with user testing [23, 24]. Due to its flexible nature of application, heuristic evaluation has been adapted to a range of specialized domains, including game design [35] and e-commerce website design [19]. In this study, heuristic evaluation was adapted to evaluating online MC toolkit design.

Sandboxr is an online 3D MC toolkit allowing consumers to create customized 3D printed figures of video game characters. It applies a modularity approach which allows consumers to select different parts, i.e. modules of the product and assemble them to design a final product. Nervous System follows a generative approach to customization which creates computer simulations or algorithms to generate designs.

Sandboxr provides option lists that allow consumers to choose the design they want. In particular, all the options in Sandboxr are predetermined by designers or

\footnotetext{
${ }^{1}$ https://sandboxr.com/.

${ }^{2}$ http://n-e-r-v-o-u-s.com/.
} 
manufacturers, which means designers or manufacturers retain the ultimate control over the design of the product. In this case, the size of the solution space is limited by the number of modules that designers or manufacturers allow consumers to customize and the options they provide.

Nervous System on the contrary offers more design freedom and creativity to consumers. Nervous System provides a set of tools that allow consumers to directly manipulate and modify the structure of the 3D model in real time. However, the generative approach taken by Nervous System means that each of their designs is the output of a computer program. Each application on the Nervous System website therefore needs programmers to write code for their implementation, which is not easy or flexible for generating more designs in a short time.

Regarding the interaction design, the two toolkits follow some of the guidelines identified in Table 1, but also fail to follow others. Instead of letting consumers design from scratch, both toolkits provide a preset design as the starting point. This helps to make the process more understandable and accessible for consumers. Sandboxr employs a predesigned customization procedure, i.e. consumers have to follow a strict order to customize a game figure. A timeline is used to tell consumers which step they are in and how many steps remain until completion. Nervous System provides a flexible design procedure, but it does not provide enough information to let consumers know which step they are in. Therefore, it is easier for consumers to get lost especially considering it has a vast solution space. Sandboxr does not allow consumers to save unfinished designs, and it provides no way for consumers to compare different design ideas. On Nervous System website, consumers can name their design and save it to a database at any time. They can return later and edit the saved design to complete the order. In this case, Nervous System provides consumers with more support for their creativities.

The direct manipulation of the 3D model on Sandboxr is limited. Consumers can only rotate the 3D model but not zoom in/out or change the structure of the 3D model. After consumers make the decision at each step, it takes a while for the toolkit to show the outcome. Nervous System gives more freedom to consumers. It allows consumers to directly manipulate the 3D model and modify its shape and structure. Feedback of the consumer's customization changes is instant which gives consumers a strong sense of control.

The limitation of consumer interaction to a large extent is because of the 3D modelling technologies the toolkit employs. Sandboxr employs traditional textured polygonal meshes as their geometric model representation. The model parameterization is limited to size selection. Nervous System uses polygonal meshes to represent geometric models. It provides a very high level of parameterization. Each model has to be generated from scratch by generative modelling software tools rather than traditional CAD design tools. Here, the designer has to closely work with or to be a software developer to properly implement the generative procedures in software. Nervous System is also a very advanced toolkit from the web technology point of view, incorporating several scripting languages and WebGL.

Despite providing an adaptive approach to toolkit design ensures the usability and accessibility of toolkits for consumers with different skills, experience and 
knowledge, neither Nervous System nor Sandboxr considers individual differences among the actual consumer group. However, mass customization is currently still at an early stage of development. Technical limitations and the lack of proper design strategies mean that an adaptive approach to toolkit design is currently still just a theory. It is expected that future toolkits will meet different consumer's needs and requirements.

\section{Conclusion and Future Work}

In this paper, we have provided an overview of existing studies in MC toolkit design, giving particular focus to online 3D MC toolkits and the consumers' interactions with the toolkits. Based on this review, we have proposed an evaluation model, which attempts to provide a comprehensive understanding of 3D toolkit design from four aspects: consumer, solution space, consumer-toolkit interaction and technological support. Specifically, individual difference, solution space, interaction design, 3D modelling technology and web technology constitute the key dimensions for evaluating 3D MC toolkits. Despite a fair amount of efforts that have been devoted to theoretical research, current 3D toolkits are still at an early development stage, and a number of research questions need to be addressed as directions for future research.

Previous research has found that individual differences in knowledge, skill, creative talent and previous experience require different design strategies for solution space design and interaction design. Despite a number of studies suggesting how to adapt toolkit design to different consumers, few efforts have actually been made to apply them into practice. Therefore, research questions that we suggest should be considered in terms of individual differences include:

- In addition to knowledge, skill, creative talent and previous experience, what other individual factors would influence toolkit design? For example, gender? Age? Income?

- What is the best way to discover individual differences, especially for new customers? An explicit approach, for example, could directly ask the consumer's gender or preferences or an implicit approach that the toolkit adapts itself to consumer capabilities without letting them know.

- Given a set of identified differences, how should the toolkit adapt itself to best suit these?

Solution space design is understood as all the possible designs a toolkit can provide. Specifically, the size of the solution space and the types of options are two main concerns. Here we argue that the size of the solution space is not a single dimension. It is influenced by a number of factors, such as the type of product and the approaches of customization. For example, the number of options for customizing the aesthetic aspect of a product may be different from the number of options for customizing the functional aspect of a product because the function 
of a product may be restricted from a practical purpose as well as safety or legal concerns. Given these considerations, we suggest that future research questions which should be considered for solution space design include:

- What are the factors that influence the size of the solution space?

- What are the efficient ways of organizing options for different customization approaches, i.e. veneer, modularity, parametric and generative approaches, to avoid mass confusion?

- What auxiliary information should be provided to help consumers understand each customizable option, e.g. how to interact with it and what effects it will bring to customizing the product?

- Considering individual differences, what is the best way to adapt the solution space design to different consumer's needs and preferences?

Interaction design refers to the process of the consumer interacting with the toolkits and customizing the product. Most MC toolkits are lacking in basic $\mathrm{HCI}$ principles [44]. We suggest the following research questions for future research in interaction design for MC:

- What HCI principles should be followed by mass customization toolkit design?

- What support can be provided to the consumers to help them understand the process of interaction and customizing the product?

- What are the effective ways to encourage consumer's interaction and creativity while customizing the product?

- Considering individual differences, what different interactive strategies can be applied to difference consumers or consumer groups?

The design of a toolkit is closely related to technical development. In particular, $3 \mathrm{D}$ toolkit design is a special area which is quite different from $2 \mathrm{D}$ toolkit design in terms of the visual representation of products and the way consumers interact with the toolkits. Therefore, 3D toolkits bring different experiences to consumers when compared to 2D toolkits, which also have higher requirements for the technical support, especially for the development of 3D modelling and web technologies. Different technologies bring different capabilities and restrictions to toolkit design, many of which can only be improved by technical breakthroughs in the future. Therefore, future research questions in terms of enabling technologies include:

- What are the most intuitive interface elements for an MC model and how can they be supported by the modelling representation scheme? From a technical standpoint, one of the easiest ways to technically provide access to a model's parameters is through slider interface elements, but this is not necessarily the most intuitive from the user's standpoint.

- How can MC interfaces make better use of established and emerging technologies and portable devices, including touchscreen, gestures or VR?

- How could a collaborative interface be presented for MC, reflecting requirements and views of different audiences? This would allow a professional designer and client to look at the same artefact but see them in different ways and interact with them through a different interface in different ways. 
- Given recent advances in multimaterial 3D printing hardware and supporting model representation schemes, how can a viable and intuitive interface be made for multimaterial products and how can these be made available for MC?

This study helps us understand the current state of MC research especially in terms of toolkit design in academia. However, most current studies take a theoretical approach rather than an empirical approach to propose their research findings. In other words, researchers draw their conclusions based on their knowledge in related areas (e.g. HCI, psychology, etc.) or their analysis of a few online MC toolkits rather than actually observing consumers using toolkits to customize a product or testing their findings on consumers. Therefore, in the future, we expect to conduct user studies to test what we find in this study and conduct empirical studies to discover the answers to the research questions we proposed above. In the end, we hope to construct comprehensive and systematic design guides for online 3D mass customization toolkit design.

\section{References}

1. Abdallah, A., Fryazinov, O., Adzhiev, V., Pasko, A.: 3D web-based shape modelling: building up an adaptive architecture. ACHI 2014: The Seventh International Conference on Advances in Computer-Human Interactions, Barcelona, Spain, pp. 96-102 (2014)

2. Bandura, A.: Self-efficacy: the exercise of control, Worth Publishers, New York (1997)

3. Boese, E.S.: An introduction to programming with Java applets. Jones \& Bartlett Publishers, Boston (2009)

4. Blake, B.F., Neuendorf, K.A., Valdiserri, C.M.: Tailoring new websites to appeal to those most likely to shop online. Technovation. 25(10), 1205-1214 (2005)

5. Chen, J., Shapiro, V., Suresh, K., Tsukanov, I.: Shape optimization with topological changes and parametric control. Int. J. Numer. Methods Eng. 71(3), 313-346 (2007)

6. Daugherty, T., Li, H., Biocca, F.: Consumer learning and 3-D ecommerce: the effects of sequential exposure of a virtual experience relative to indirect and direct product experience on product knowledge, brand attitude and purchase intention. Unpublished Dissertation, Michigan State University, East Lansing, (2001)

7. Gandhi, A., Magar, C., Roberts, R.: How technology can drive the next wave of mass customization. Business Technology Office 1-8 (2014). https://www.mckinsey.com/ /media/ mckinsey/dotcom/client_service/bto/pdf/mobt32_02-09_masscustom_r4.ashx

8. Fogliatto, F.S., da Silveira, G.J., Borenstein, D.: The mass customization decade:an updated review of the literature. Int. J. Prod. Econ. 138(1), 14-25 (2012)

9. Franke, N., Hader, C.: Mass or only "niche customization"? Why we should interpret configuration toolkits as learning instruments. J. Prod. Innov. Manag. 31(6), 1214-1234 (2014)

10. Franke, N., Piller, F.T.: Key research issues in user interaction with user toolkits in a mass customization system. Int. J. Technol. Manag. 26(5-6), 578-599 (2003)

11. Franke, N., Piller, F.: Toolkits of user innovation and design: an exploration of user interaction and value creation in the watch market. Proceedings of the 2nd Interdisciplinary World Congress on Mass Customization and Personalization, Munich, Germany (2003)

12. Franke, N., Shah, S.: How communities support innovative activities: an exploration of assistance and sharing among end-users. Res. Policy. 32(1), 157-178 (2003)

13. Füller, J., Matzler, K., Hutter, K., Hautz, J.: Consumers' creative talent: which characteristics qualify consumers for open innovation projects? An exploration of asymmetrical effects. Creativity Innov. Manage. 21(3), 247-262 (2012) 
14. Gerber, E.M., Martin, C.K.: Supporting creativity within web-based selfservices. Int. J. Design. 6(1), 85-100 (2012)

15. Hermans, G.: A model for evaluating the solution space of mass customization toolkits. International. J. Ind. Eng. Manage. 3(4), 205-214 (2012)

16. Hermans, G.: Identifying User-as-Designer Behaviors when Designing by Using Toolkits. Proceedings of the 10th European Academy of Design Conference. University of Gothenburg, Gothenburg (2013)

17. Hoffmann, C.M.: Geometric and Solid Modeling. Morgan Kaufmann Pub, San Mateo (1998)

18. Huang, Z., Benyoucef, M.: From e-commerce to social commerce: a close look at design features. Electron. Commer. Res. Appl. 12(4), 246-259 (2013)

19. Huffman, C., Kahn, B.E.: Variety for sale: mass customization or mass confusion? J. Retail. 74(4), 491-513 (1998)

20. Hunt, D.M., Radford, S.K., Evans, K.R.: Individual differences in consumer value for mass customized products. J. Consum. Behav. 12(4), 327-336 (2013)

21. Lin, C.H., Hsu, Y.L., Sun, T.H.: The application of 3D interactive media on mass customization design of hardwood furniture. Proceedings of International Conference on Planning and Design, Taiwan (2003)

22. Jeffries, R., Miller, J.R., Wharton, C., Uyeda, K.: User interface evaluation in the real world: a comparison of four techniques. In Proceedings of the SIGCHI conference on Human factors in computing systems, pp. 119-124. ACM, New Orleans (1991)

23. Karat, C.M., Campbell, R., Fiegel, T.: Comparison of empirical testing and walkthrough methods in user interface evaluation. In Proceedings of the SIGCHI conference on Human factors in computing systems, pp. 397-404. ACM, Monterey (1992)

24. Khalid, H.M., Helander, M.G.: Web-based do-it-yourself product design. In: The Customer Centric Enterprise, pp. 247-266. Springer, Berlin/Heidelberg (2003)

25. Khalid, H.M., Oon, Y.B.: Usability of configuration systems in design by customer web sites. Proceedings of the Human Factors and Ergonomics Society Annual Meeting, pp. 811-815. SAGE Publications (2003)

26. Lüthje, C.: Characteristics of innovating users in a consumer goods field: an empirical study of sport-related product consumers. Technovation. 24(9), 683-695 (2004)

27. Maes, P.: Agents that reduce work and information overload. Commun. ACM. 37(7), 30-40 (1994)

28. Matzler, K., Stieger, D., Füller, J.: Consumer confusion in internet-based mass customization: testing a network of antecedents and consequences. J. Consum. Policy. 34(2), 231-247 (2011)

29. Ng, Y.M.: Customizable 3D virtual objects: a breakthrough in electronic catalogs in Internet business. MPhil thesis, The Hong Kong University of Science and Technology, Hong Kong (2000)

30. Nichols, S., Haldane, C., Wilson, J.R.: Measurement of presence and its consequences in virtual environments. Int. J. Hum. Comput. Stud. 52(3), 471-491 (2000)

31. Nielsen, J.: Usability inspection methods. In Conference companion on Human factors in computing systems, pp. 413-414, ACM (1994)

32. Parisi, T.: Programming 3D Applications with HTML5 and WebGL: 3D Animation and Visualization for Web Pages. O'Reilly Media Inc, Beijing (2014)

33. Piller, F., Schubert, P., Koch, M., Möslein, K.: Overcoming mass confusion: collaborative customer co- design in online communities. J. Comput.-Mediat. Commun. 10(4), 00 (2005)

34. Pinelle, D., Wong, N., Stach, T.: Heuristic evaluation for games: usability principles for video game design. In Proceedings of the SIGCHI Conference on Human Factors in Computing Systems, pp. 1453-1462, ACM (2008)

35. Randall, T., Terwiesch, C., Ulrich, K.T.: Research note-user design of customized products. Mark. Sci. 26(2), 268-280 (2007)

36. Salvador, F., De Holan, P.M., Piller, F.: Cracking the code of mass customization. MIT Sloan Manag. Rev. 50(3), 71-78 (2009)

37. Schnurr, B., Scholl-Grissemann, U.: Beauty or function? How different mass customization toolkits affect customers' process enjoyment. J. Consum. Behav. 14(5), 335-343 (2015) 
38. Shapiro, V., Vossler, D.L.: What is a parametric family of solids? Proceedings of the third ACM symposium on Solid modeling and applications, pp. 43-54, ACM (1995)

39. Thau, D.: The Book of JavaScript: A Practical guide to interactive Web pages. No Starch Press, San Francisco (2006)

40. Totz, C., Riemer, K.: The effect of interface quality on success-an integrative approach on mass customization design. Proceedings of the 1st World Congress on Mass Customization and Personalization, Hong-Kong (2001)

41. Vilbrandt, T., Fryazinov, O., Stamm, C., Pasko, A.: A web oriented function-based volume modeling framework. Technical Report TR-NCCA-2008-02, Bournemouth University, Bournemouth, UK (2008)

42. Von Hippel, E.: User toolkits for innovation. J. Prod. Innov. Manag. 18(4), 247-257 (2001)

43. Von Neumann, J.: Can we Survive Technology? Fortune 91(6), 106 (1955)

44. Walcher, D., Piller, F. T.: The customization 500: an international benchmark study on mass customization and personalization in consumer e-commerce. ICON Group International (2012). http://www.lulu.com/shop/dominik-walcher-and-frank-piller/the-customization-500ebook-retail/ebook/product-20869715.html

45. West, M.A., Farr, J.L. (eds.): Innovation and Creativity at Work: Psychological and Organizational Strategies. Wiley, Chichester (1990)

46. Westland, J.C., Au, G.: A comparison of shopping experiences across three competing digital retailing interfaces. Int. J. Electron. Commer. 2(2), 57-69 (1997)

47. Wikström, S.: Value creation by company- consumer interaction. J. Mark. Manag. 12(5), 359-374 (1996)

Open Access This chapter is licensed under the terms of the Creative Commons Attribution 4.0 International License (http://creativecommons.org/licenses/by/4.0/), which permits use, sharing, adaptation, distribution and reproduction in any medium or format, as long as you give appropriate credit to the original author(s) and the source, provide a link to the Creative Commons license and indicate if changes were made.

The images or other third party material in this chapter are included in the chapter's Creative Commons license, unless indicated otherwise in a credit line to the material. If material is not included in the chapter's Creative Commons license and your intended use is not permitted by statutory regulation or exceeds the permitted use, you will need to obtain permission directly from the copyright holder. 